\title{
Induction of osteoclastogenesis and bone loss by human autoantibodies against citrullinated vimentin
}

\author{
Ulrike Harre, ${ }^{1}$ Dan Georgess, ${ }^{2}$ Holger Bang, ${ }^{3}$ Aline Bozec, ${ }^{1}$ Roland Axmann, ${ }^{1}$ Elena Ossipova, ${ }^{4}$ \\ Per-Johan Jakobsson, ${ }^{4}$ Wolfgang Baum, ${ }^{1}$ Falk Nimmerjahn, ${ }^{5}$ Eszter Szarka, ${ }^{6}$ Gabriella Sarmay, ${ }^{6}$ \\ Grit Krumbholz, ${ }^{7}$ Elena Neumann, ${ }^{7}$ Rene Toes, ${ }^{8}$ Hans-Ulrich Scherer, ${ }^{8}$ Anca Irinel Catrina, ${ }^{3}$ \\ Lars Klareskog, ${ }^{3}$ Pierre Jurdic, ${ }^{2}$ and Georg Schett ${ }^{1}$

\begin{abstract}
'Department of Internal Medicine 3, University of Erlangen-Nuremberg, Erlangen, Germany. ²Institut de Génomique Fonctionnelle de Lyon, Université de Lyon, and CNRS, Ecole Normale Superieure de Lyon, Lyon, France. ${ }^{3}$ Orgentec Diagnostika, Mainz, Germany. ${ }^{4}$ Rheumatology Unit, Department of Medicine, Karolinska Institute and Karolinska University Hospital, Stockholm, Sweden. ${ }^{5}$ Department of Genetics, University of Erlangen-Nuremberg, Erlangen, Germany. ${ }^{6}$ Department of Immunology, Eotvos Lorand University, Budapest, Hungary. ${ }^{7}$ Department of Rheumatology, University of Giessen, Bad Nauheim, Germany. ${ }^{8}$ Department of Rheumatology, Leiden University Medical Center, Leiden, Netherlands.
\end{abstract}

\begin{abstract}
Autoimmunity is complicated by bone loss. In human rheumatoid arthritis (RA), the most severe inflammatory joint disease, autoantibodies against citrullinated proteins are among the strongest risk factors for bone destruction. We therefore hypothesized that these autoantibodies directly influence bone metabolism. Here, we found a strong and specific association between autoantibodies against citrullinated proteins and serum markers for osteoclast-mediated bone resorption in RA patients. Moreover, human osteoclasts expressed enzymes eliciting protein citrullination, and specific $\mathbf{N}$-terminal citrullination of vimentin was induced during osteoclast differentiation. Affinity-purified human autoantibodies against mutated citrullinated vimentin (MCV) not only bound to osteoclast surfaces, but also led to robust induction of osteoclastogenesis and boneresorptive activity. Adoptive transfer of purified human MCV autoantibodies into mice induced osteopenia and increased osteoclastogenesis. This effect was based on the inducible release of TNF- $\alpha$ from osteoclast precursors and the subsequent increase of osteoclast precursor cell numbers with enhanced expression of activation and growth factor receptors. Our data thus suggest that autoantibody formation in response to citrullinated vimentin directly induces bone loss, providing a link between the adaptive immune system and bone.
\end{abstract}

\section{Introduction}

RA affects about $1 \%$ of the population worldwide and is one of the most destructive diseases in humans $(1,2)$. Current concepts suggest that RA emerges by complex gene-environment interactions involving HLA and other genes, as well as in response to environmental factors $(3,4)$. Smoking, for instance, elicits cellular responses, such as posttranslational modification of proteins; among these, citrullination is considered a key stress response of cells to noxious stimuli. Citrullination is achieved by a group of enzymes called peptidylarginine deiminases (PADs), which metabolize the amino acid arginine into citrulline (5). Smoking induces citrullination of the bronchial epithelium. In addition, bacteria involved in periodontitis, such as Porphyromonas gingivalis, also strongly induce protein citrullination $(3,6)$. Interestingly, more than $70 \%$ of patients with RA show a breach in tolerance for citrullinated proteins eliciting an anti-citrullinated protein antibody (ACPA) response $(7,8)$. This ACPA response is highly specific for RA and not found in other forms of autoimmune disease. The formation of ACPAs is closely related to certain HLA genotypes, like the shared epitope and environmental factors (such as smoking). Interestingly, ACPAs emerge years before the onset of clinically overt RA, which suggests that autoimmunity precedes inflammation in RA (9).

Authorship note: Ulrike Harre and Dan Georgess contributed equally to this work. Conflict of interest: Holger Bang is an employee of Orgentec Diagnostika. Citation for this article: J Clin Invest. 2012;122(5):1791-1802. doi:10.1172/JCI60975.
It has always been stunning how effectively RA destroys bone. If the inflammatory disease process is not treated early and effectively, then bone erosions emerge after a few months of disease and lead to the destruction of the entire joint (10). Moreover, osteoporosis emerges fast in RA and affects even patients with early disease, leading to an increased fracture risk later on. Inflammation appears to be a key risk factor for bone loss in RA. Surrogate markers for high inflammatory disease activity, such as high C-reactive protein levels, are predictive for local and systemic bone loss (11). The negative influence of inflammation on bone reflects the tight interactions between the immune system and the skeleton, which have recently lead to a new research field termed osteoimmunology (12). Apart from inflammatory disease burden, however, the strongest risk factor for bone loss is the presence of ACPAs. Several studies have shown that ACPAs are among the strongest predictors for bone-erosive disease in RA, strongly suggesting a direct link between autoantibody response in RA and the ability of the disease to elicit structural bone damage (13-16). We therefore hypothesized that the main autoantibody response in RA, namely that of ACPAs, directly influences bone homeostasis. As bone resorption is considered to be among the leading mechanisms of bone loss in RA, we studied whether ACPAs influence bone resorption.

\section{Results}

ACPAs are associated with increased bone resorption in patients with $R A$. Given that the presence of ACPAs in RA patients is associated with bone loss and our hypothesis that ACPAs themselves can precipitate bone loss by influencing bone metabolism, we first sought to 

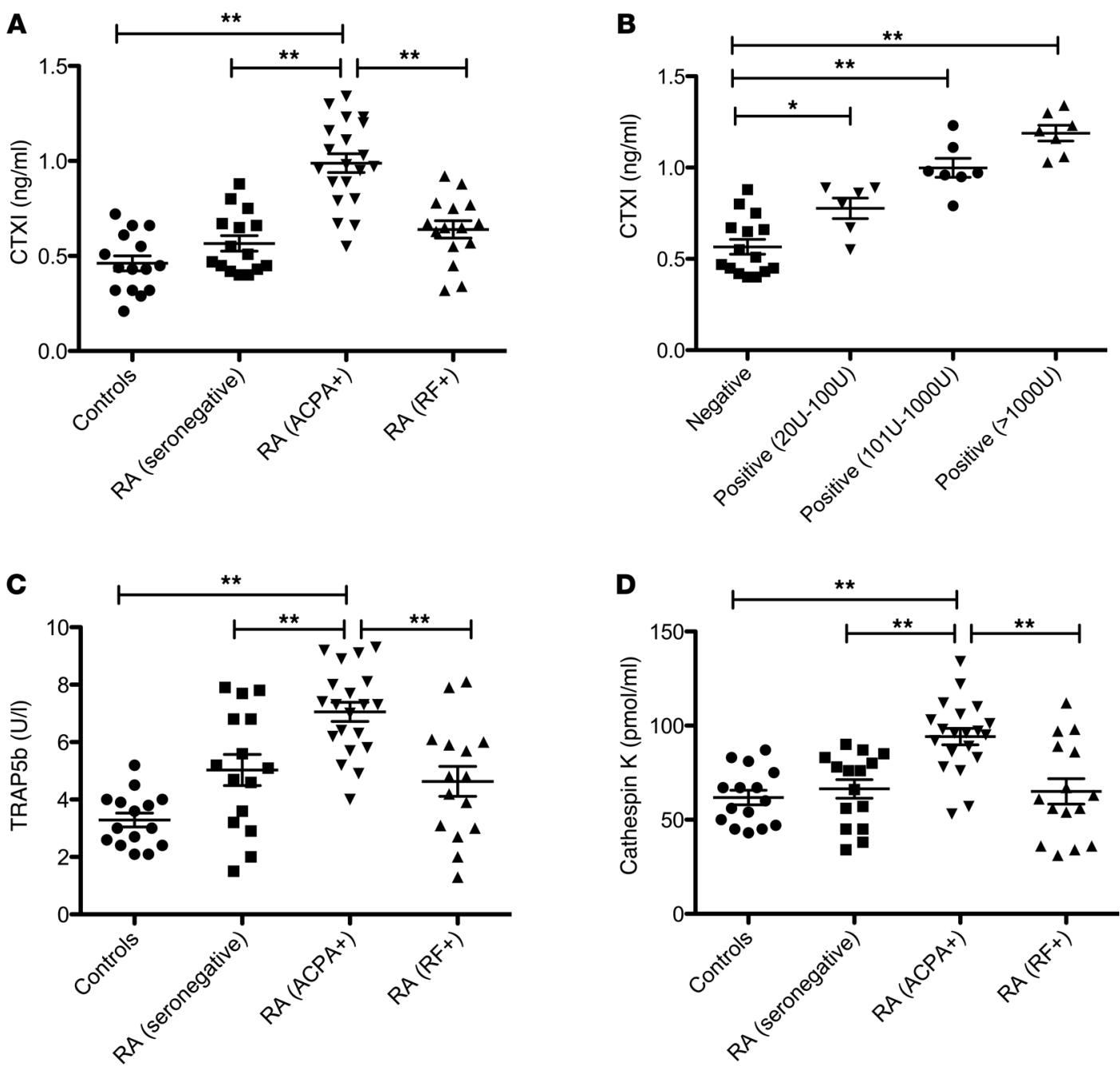

Figure 1

ACPAs are linked to high bone resorption in humans. Serum samples obtained from healthy controls as well as patients with RA without ACPAs or with positivity for rheumatoid factor (RF) or ACPAs were investigated for the bone resorption parameters CTXI (A and B), TRAP5b (C), and cathepsin $\mathrm{K}(\mathbf{D})$. In $\mathbf{B}$, different ACPA responses are shown (expressed in units). ${ }^{*} P<0.05 ;{ }^{* *} P<0.01$.

determine whether the presence of ACPAs in RA patients is linked to altered bone metabolism. We investigated serum samples from RA patients with ACPAs for markers of bone resorption and bone formation and compared the results with those of RA patients without ACPAs as well as normal controls. To minimize bias, we focused on newly diagnosed RA patients without concomitant treatment of glucocorticoids or other immunosuppressants. We also carefully matched the groups (ACPA-positive/rheumatoid factor-negative RA, ACPA-negative/rheumatoid factor-positive RA, ACPA-negative/rheumatoid factor-negative RA, and healthy controls) for age and sex as well as for disease activity and disease duration. When analyzing C-terminal cleavage products for collagen type I (CTXI) as a marker of bone resorption, we found significantly higher CTXI in ACPA-positive patients than in all other groups $(P<0.01$; Figure $1 \mathrm{~A})$. The influence of ACPA on bone resorption in RA patients was even more evident when ACPA titers were correlated with CTXI levels. Even patients with low ACPA levels $(<200 \mathrm{U} / \mathrm{ml})$ had higher CTXI than did RA patients without ACPA (Figure 1B). Moreover, CTXI level was related to ACPA titer, as patients with modest and high
ACPA levels showed a dose-dependent increase of bone resorption (Figure 1B). Similar results were observed with other markers of bone resorption, such as the serum level of the osteoclast-derived enzymes tartrate-resistant acid phosphatase 5b (TRAP5b) and cathepsin K: RA patients with ACPA showed significantly higher levels of these markers (Figure 1, C and D). In contrast, bone formation, which was measured by bone alkaline phosphatase, was the same among RA patients with or without ACPA, with levels tending toward lower than those of healthy controls. These data support the notion that the presence of ACPA is associated with bone loss in RA.

Isolation of ACPAs with specificity to mutated citrullinated vimentin from buman RA patients. We consequently sought to determine the mechanism by which ACPAs are linked to enhanced bone resorption, hypothesizing that these autoantibodies directly stimulate the differentiation of bone-resorbing cells. To test this concept, we first had to exactly define the ACPA responses in human RA patients that are strongly linked to bone resorption. We therefore affinity-purified ACPAs from 3 individual RA patients with very high ACPA responses (>1,000 $\mathrm{U}$ ) and high levels of bone resorp- 

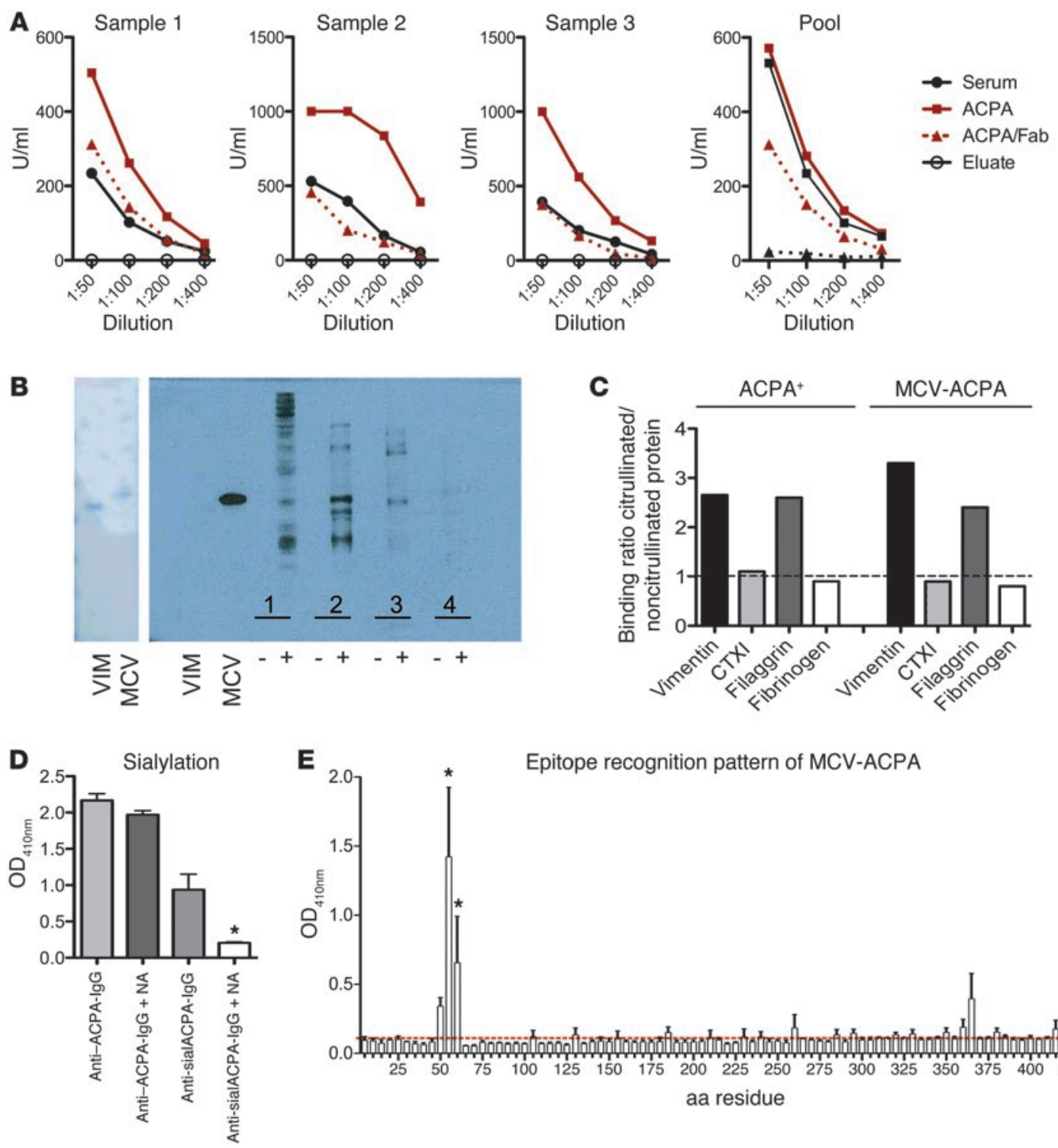

Figure 2

Isolation of ACPAs from human serum. (A) ACPA reactivity of the original serum from RA patients, purified ACPAs, the corresponding Fab fragment (ACPA/Fab), and the remaining eluated IgG fraction (eluate) for 3 individual serum samples as well as the pooled samples. Dotted curves in the graph of the pooled sample indicate detection with an Fc- specific antibody, with loss of reactivity of the Fab fraction confirming its purity. (B) Coomassie gel and Western blot of HeLa cells showing the binding of purified antibodies against vimentin (VIM), MCV, and cytosolic (1), membrane (2), nuclear (3) and actin-containing cytoskeletal (4) cell factions before (-) and after (+) treatment with PAD. (C) ELISA showing reactivity of ACPAs and MCV-ACPAs against noncitrullinated and citrullinated peptides of vimentin, fibrinogen, and CTXI. $y$ axis shows the binding ratio with specificity to citrullinated peptides giving values higher than 1 (dotted line). (D) Sialylation of MCV-ACPAs was analyzed by assessing the S2 glycoform (anti-sial ACPA) by ELISA before and after neuraminidase (NA) treatment. (E) Epitope reaction pattern of MCV-ACPAs was analyzed by assessing binding to 25 -mer peptides spanning the entire sequence of citrullinated vimentin. $\mathrm{OD}_{410 \mathrm{~nm}}, \mathrm{OD}$ at a wavelength of $410 \mathrm{~nm}$. ${ }^{\star} P<0.05$.

tion. We decided to isolate human ACPAs with specificity to mutated citrullinated vimentin (MCV), as these autoantibodies are highly specific for RA $(17,18)$, and vimentin is expressed by mononuclear cells and osteoclasts (19). By using a CNBr-sepharose column loaded with MCV, we were able to purify ACPAs and retain - and even enrich - their strong reactivity to MCV (Figure 2A). Moreover, we also cleaved the affinity-purified ACPAs with pepsin to receive $\mathrm{F}\left(\mathrm{ab}^{\prime}\right) 2$ fragments, which also retained high reactivity against MCV but lacked the Fc portion (Figure 2A).
Western blot analysis of the isolated affinity-purified human ACPAs against MCV (referred to herein as MCV-ACPAs) on naive and PAD-treated HeLa cells showed exclusive specificity to citrullinated vimentin, but not to noncitrullinated vimentin, after PAD treatment (Figure 2B). When analyzing binding to citrullinated and noncitrullinated protein extracts from different subcellular compartments, we observed that MCV-ACPAs particularly bound to the membrane fraction and, to a lesser extent, to the nuclear and cytosolic fractions, but exhibited virtually no binding to the 
A

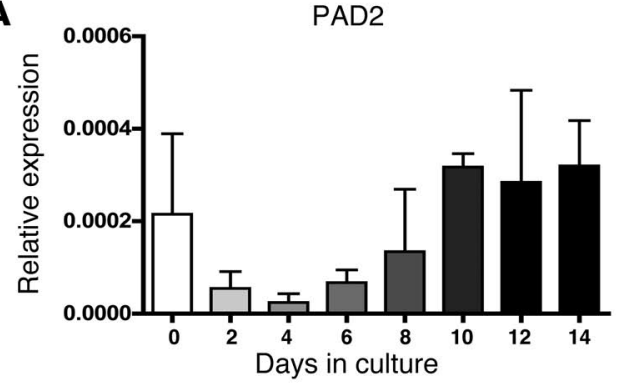

C

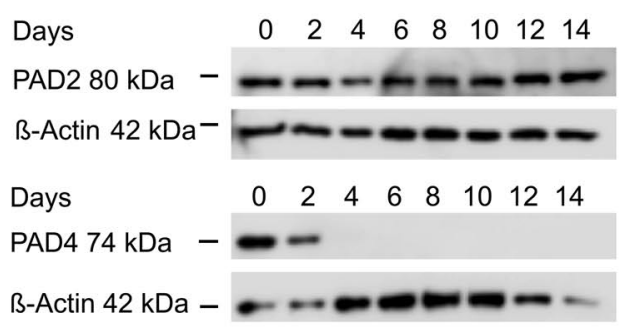

E

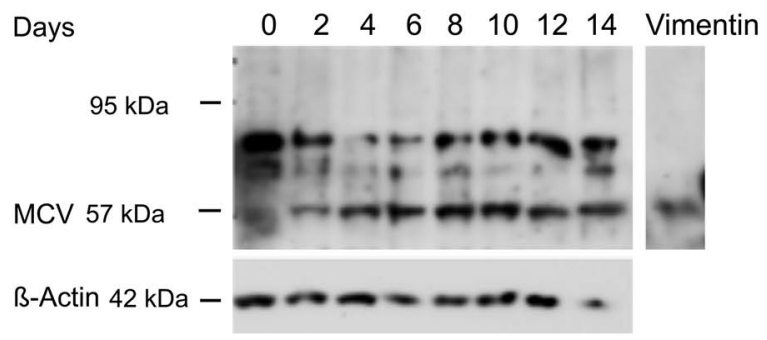

B

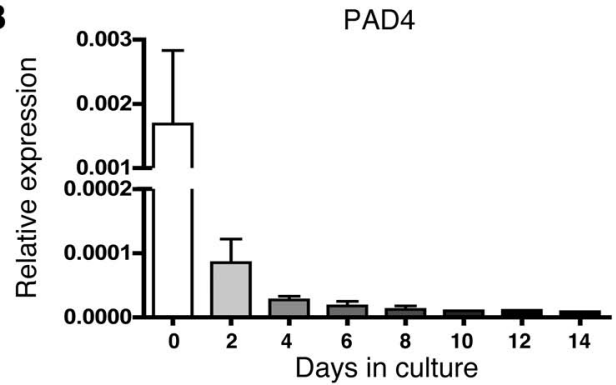

D

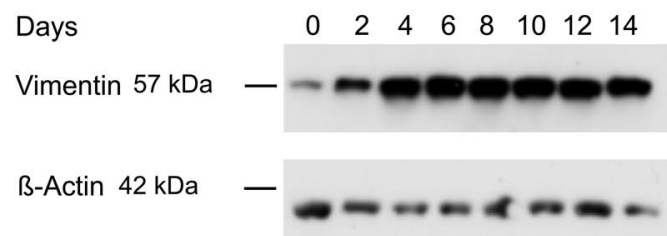

$\mathbf{F}$

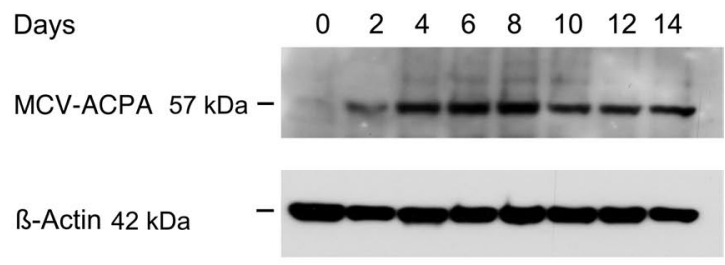

\section{Figure 3}

Expression of PAD enzyme and citrullinated vimentin in osteoclasts. (A and B) Real-time PCR for PAD2 (A) and PAD4 (B) of human osteoclast precursor cells stimulated with MCSF and RANKL to achieve osteoclast differentiation. (C) Western blotting showing protein expression of PAD4 and PAD2 at various stages of osteoclast differentiation. (D) Western blotting showing protein expression of vimentin at various stages of osteoclast differentiation ( $E$ and $\mathbf{F}$ ) Western blotting showing MCV expression using a chicken antibody against citrullinated vimentin ( $E$; recombinant vimentin used as positive control) and MCV-ACPAs (F). For control purposes, staining for $\beta$-actin was performed. Representative blots from 3 independent experiments are shown.

actin-containing cytoskeletal compartment (Figure 2B). Moreover, MCV-ACPAs not only showed specificity to peptides from citrullinated vimentin, in contrast to peptides from noncitrullinated vimentin, but also did not recognize citrullinated peptides from other proteins, like CTXI and fibrinogen (Figure 2C) as well as GFAP and neurofascin (data not shown). The cross-reaction of MCV-ACPAs was only observed with citrullinated peptides of the epithelial protein fillagrin.

Analysis of the presence of sugar moieties by enzyme-linked lectin assay showed glycosylation of MCV-ACPAs, which was sensitive to neuraminidase digestion (Figure 2D). Analysis of the epitope recognition pattern of isolated MCV-ACPAs by testing binding to a peptide library spanning the entire citrullinated vimentin protein revealed a dominant $\mathrm{N}$-terminal epitope at amino acid position 56-77 (Figure 2E).

Osteoclasts express enzymes involved in citrullination and show citrullination of vimentin. We next validated the expression of the target of MCV-ACPA in the osteoclast lineage. Citrullination of proteins depends on the presence of the enzyme PAD, which metabolizes arginine into citrullin. Among the different PAD enzymes, only PAD2 and PAD4 were expressed in the monocyte lineage and were also found in osteoclast precursor cells at the mRNA and protein levels (Figure 3, A-C). Whereas PAD4 expression decreased with further differentiation of osteoclast precursors into osteoclasts, PAD2 expression increased during this process (Figure 3C). Furthermore, vimentin expression increased during osteoclast differentiation (Figure 3D). When using a commercially available antibody against citrullinated vimentin or MCV-ACPAs, we observed that expression of citrullinated vimentin also increased during osteoclast differentiation (Figure 3, E and F), which suggests that the target for MCV-ACPAs is indeed inducibly expressed in the osteoclast lineage. These data suggested strong binding of MCV-ACPAs to cells of the osteoclast lineage, particularly to cells in more advanced osteoclastogenic differentiation state with increased citrullination of vimentin.

Finally, these data were validated by performing mass spectrometry of citrullinated proteins from various differentiation stages (days $0,2,5,8,10$, and 14 ) of osteoclast lineage cells. Only 3 proteins (vimentin, actin, and $\alpha$-2-HS-glycoprotein) showed consistent citrullination during the process of osteoclast differentiation; of these, vimentin was the only one specifically citrullinated at more advanced differentiation stages (i.e., after day 5). Moreover, the 
A
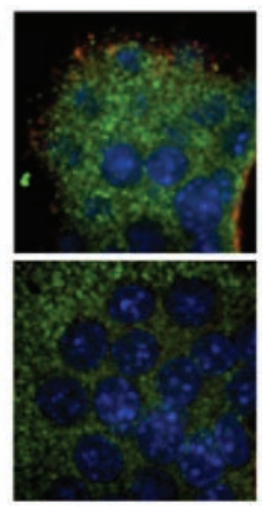
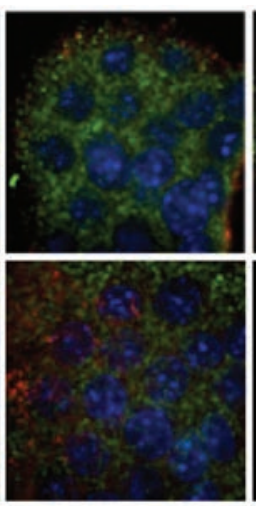
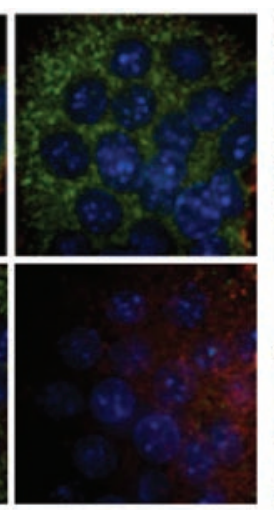
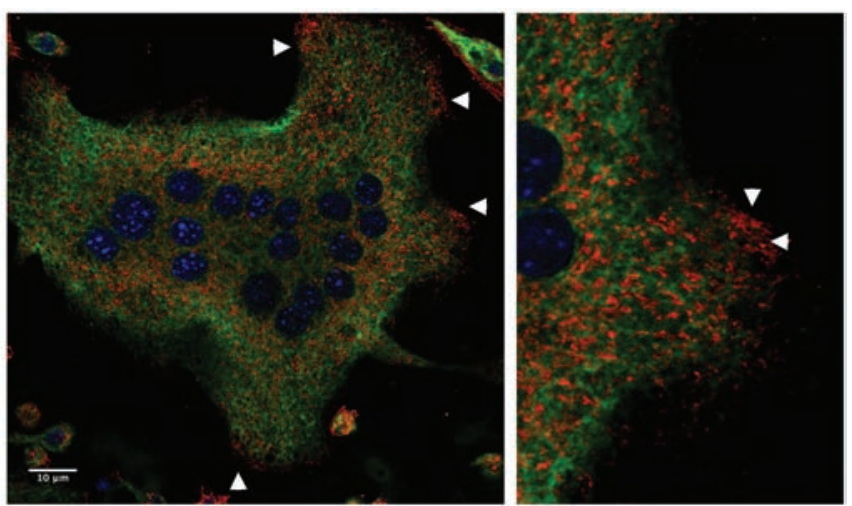

C

B

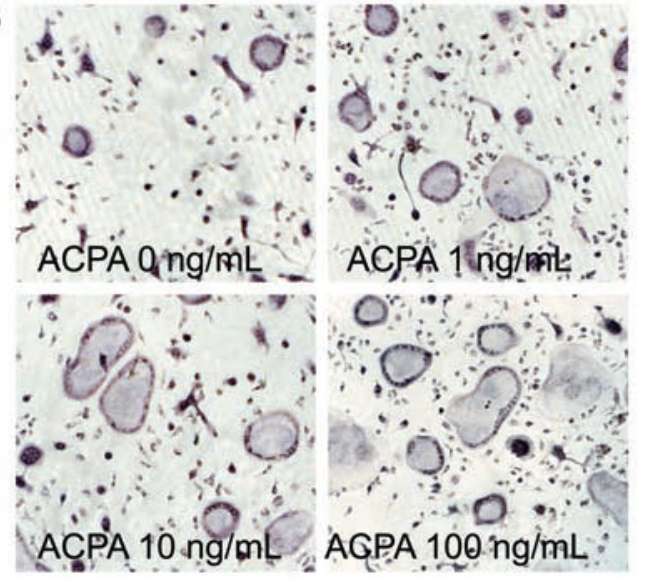

Osteoclastogenesis

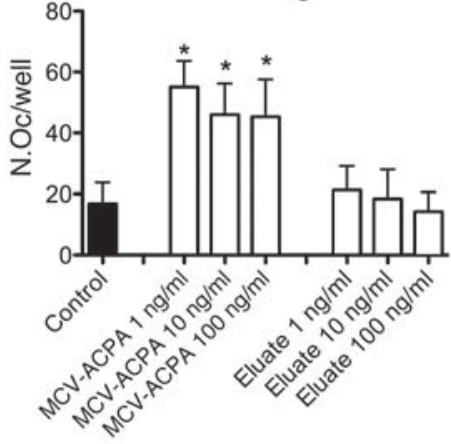

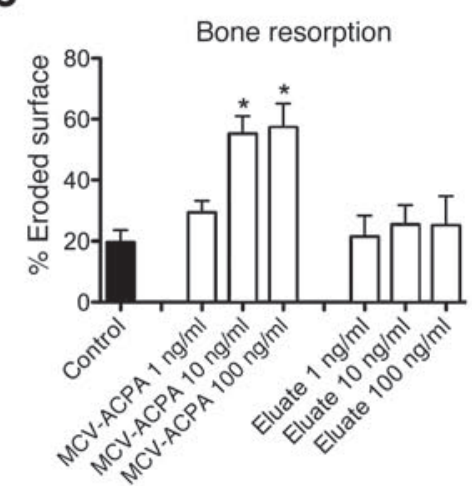

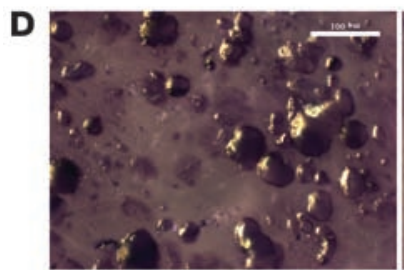

Control

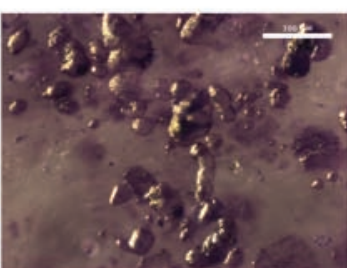

MCV-ACPA $10 \mathrm{ng} / \mathrm{ml}$

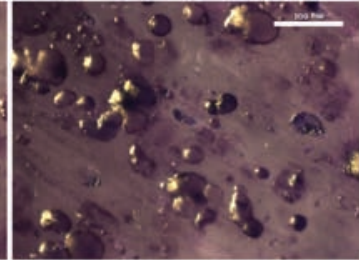

Eluate $10 \mathrm{ng} / \mathrm{ml}$

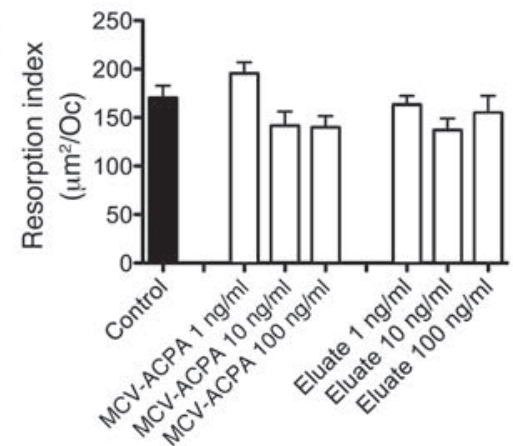

Figure 4

MCV-ACPAs stimulate osteoclastogenesis. (A) Laser scanning microscopy of 2-mm osteoclast sections showing binding of MCV-ACPAs. Left: green, MCV-ACPA staining for citrullinated vimentin; red, phalloidin staining for actin; blue, DAPI staining for the nucleus. Right: green, vimentin staining; red, MCV-ACPA staining for citrullinated vimentin; blue, DAPI staining for the nucleus. Arrowheads indicate surface staining for citrullinated vimentin. (B) Osteoclastogenesis $(n=3)$ induced by $10 \mathrm{ng} / \mathrm{ml} \mathrm{MCSF}, 1 \mathrm{ng} / \mathrm{ml}$ RANKL, and different concentrations of MCV-ACPAs and of IgG fractions deprived of ACPAs (eluate) in the presence of MCSF and RANKL. N.Oc, number of osteoclasts. (C) Resorption pit assay $(n=3)$ with different concentrations of MCV-ACPAs and of IgG fractions deprived of ACPA. (D) Assessment of resorption index $(n=3)$ by transposing a defined number of osteoclasts on bone slices and measuring the resorption pit size per single osteoclast. Control indicates no antibody addition. Original magnification, $\times 20$ (A); ×100 (A, enlarged view); ×10 (B and D). Scale bars: $100 \mu \mathrm{m} .{ }^{*} P<0.05$.

citrullinated peptide sequence of vimentin detected by mass spectrometry (KVELQELNDXFANYIDKV ${ }_{[0]}$; citrullin denoted by $\mathrm{X}$ ) was colocalized with the citrullinated epitope in the $\mathrm{N}$-terminal part of vimentin recognized by the ACPA.

$M C V$-ACPAs induce osteoclastogenesis and bone resorption. Based on our clinical observations and the experimental evidence for vimentin citrullination in osteoclasts, we hypothesized that ACPAs can directly stimulate osteoclast-mediated bone resorption. We therefore sought to determine whether ACPAs can trigger osteoclastogenesis, testing the ability of ACPA-containing serum and MCV-ACPAs to stimulate osteoclastogenesis. Using laser scanning microscopy, we found the plasma membrane and cytoplasm of osteoclasts to be stained by MCV-ACPAs (Figure 4A), indicative of citrullinated vimentin expression. Expression of citrullinated 
A

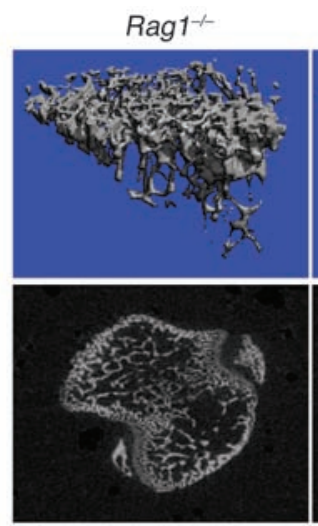

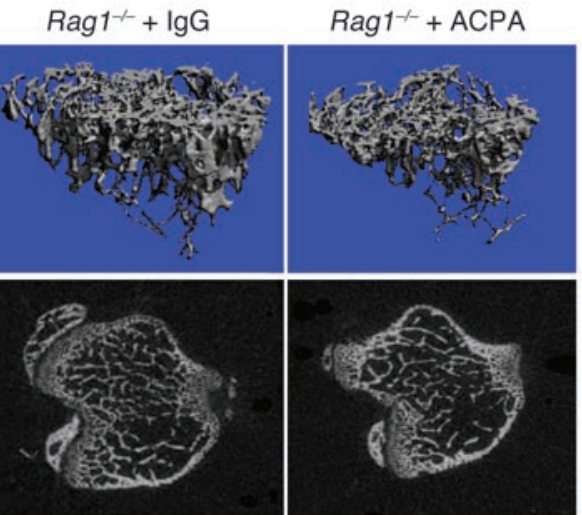

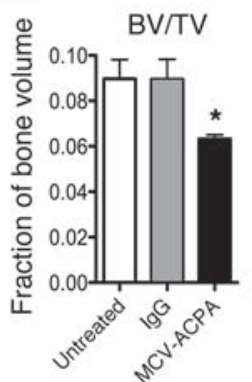

Tb.N

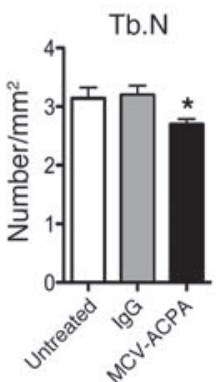

Tb.Th

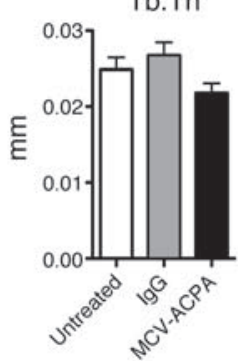

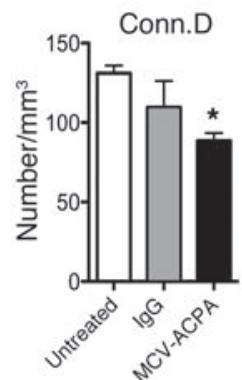
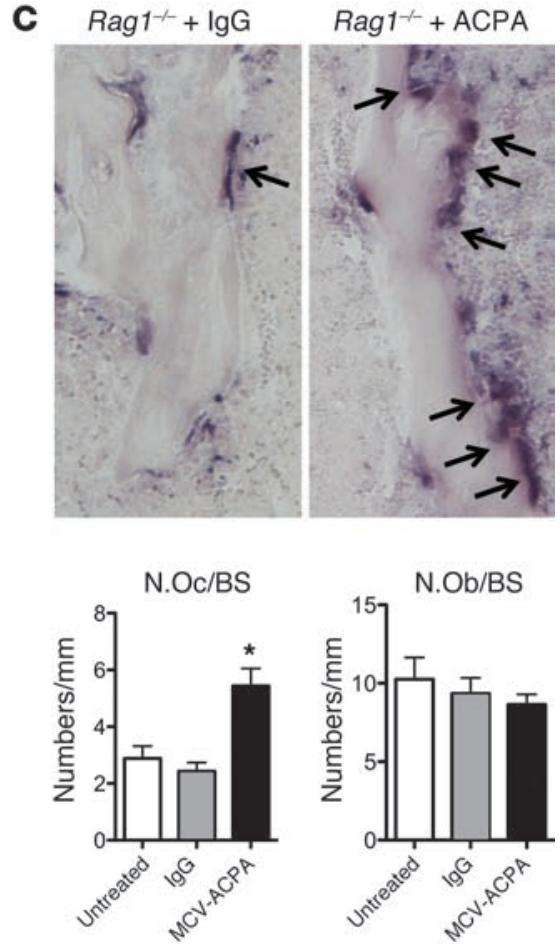

\section{Figure 5}

MCV-ACPAs induce bone loss in vivo. (A) 3-dimensional (top) and 2-dimensional (bottom) $\mu \mathrm{CT}$ images of the tibial metaphysis of Rag 1-l- mice that were left untreated or treated with control IgG or MCV-ACPAs. (B) Structural parameters of the tibial bone ( $n=5$ per group), including the ratio of bone volume to total volume (BV/TV), trabecular number (Tb.N), trabecular thickness (Tb.Th), and connectivity density (Conn.D). (C) Microphotographs (original magnification, $\times 400$ ) of tibial bones of Rag $1^{-/-}$mice treated with either IgG or MCV-ACPAs, stained for osteoclasts (purple stain and arrows) by histochemical detection of TRAP. Histomorphometric quantification of osteoclast number and osteoblast number (N.Ob) on the tibial bone surface (BS) is also shown. ${ }^{*} P<0.05$.

vimentin was not colocalized to the actin cytoskeleton, nor, interestingly, to noncitrullinated vimentin. Importantly, when performing osteoclast differentiation assays with MCSF and RANKL, we observed that addition of ACPA-containing serum (data not shown) or MCV-ACPAs increased the formation of osteoclasts (Figure 4B). In contrast, RA serum without ACPAs, as well as IgG (isolated from ACPA-positive serum samples) deprived of MCVACPAs, did not stimulate MCSF/RANKL-mediated osteoclastogenesis. Furthermore, osteoclast differentiation was enhanced by ACPAs, since TRAP-positive mononuclear cells appeared earlier in ACPA-challenged osteoclast cultures (data not shown).

When assessing bone resorption, we found a dose-dependent increase of resorption pits in the presence of ACPA-containing serum as well as MCV-ACPAs (Figure 4C), which suggests that ACPAs induce bone resorption. These findings were consistent with the use of MCV-ACPA or a Fab fragment of this antibody, excluding osteoclastogenesis stimulation and bone resorption by a solely Fc-mediated effect. Moreover, when noncitrullinated vimentin was used as an autoantigen for antibody isolation, only low amounts of antibodies could be isolated, which (a) did not recognize citrullinated vimentin and (b) did not induce osteoclastogenesis (data not shown). Furthermore, we assessed the resorption capacity of individual osteoclasts after differentiating and transposing them on bovine bone slices. These experiments showed that ACPAs did not enhance the resorptive potential of individual osteoclasts (as measured by resorption pit size of individual osteo- clasts; Figure 4D), which suggests that enhancement of overall bone resorption by ACPAs is based on enhanced differentiation and, consequently, increased numbers of osteoclasts.

MCV-ACPAs induce systemic bone loss and osteoclast formation in vivo. We next tested whether ACPAs can also induce bone loss in vivo. We challenged lymphocyte-deficient Rag1/- mice with MCV-ACPAs and control IgG, then analyzed bone structure after 4 weeks by micro-CT $(\mu \mathrm{CT})$. Analysis of bone volume relative to total volume at the tibial metaphysis showed significant reduction of trabecular bone with MCV-ACPAs compared with control IgG (Figure 5, A and B). Bone loss was based on reduced trabecular number and, consequently, lower connectivity density of trabecular bone, whereas trabecular thickness was not changed (Figure 5B). In addition, histomorphometry of trabecular bone showed a significant increase in osteoclast number along the metaphysis of ACPA-treated mice (Figure 5C), which supports the in vitro assay data and suggests that enhanced bone loss in conjunction with MCV-ACPAs is based on enhanced osteoclastogenesis and bone resorption.

Enhanced TNF production and angmentation of the osteoclast precursor cell pool as the mechanism of bone loss by MCV-ACPAs. To further elucidate the mechanism by which MCV-ACPAs induce bone loss in vivo, we examined systemic bone resorption and bone formation parameters. In accordance with the observed increase in osteoclasts in mice exposed to ACPAs, we found an increase in CTXI, a common measure of bone-resorptive activity, in the serum of ACPA- 
A

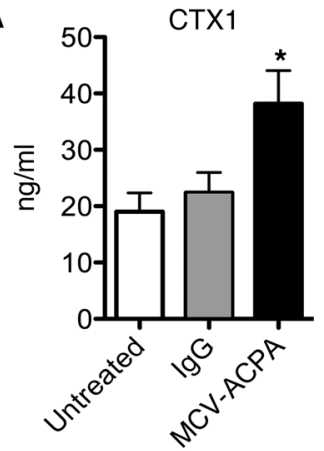

B

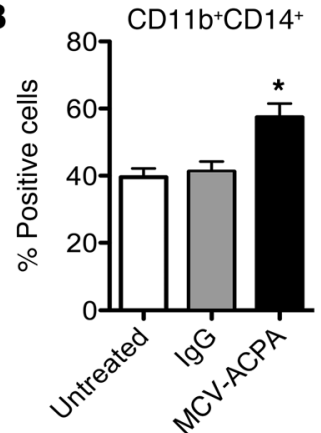

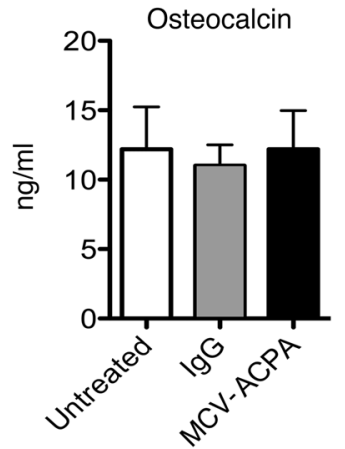

CD115

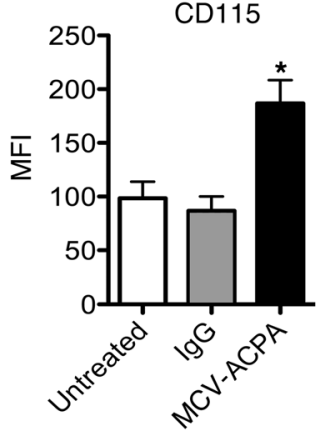

TNF- $\alpha$
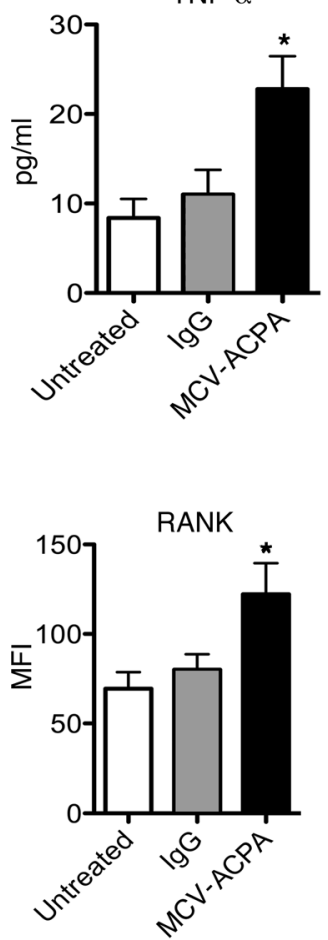

C

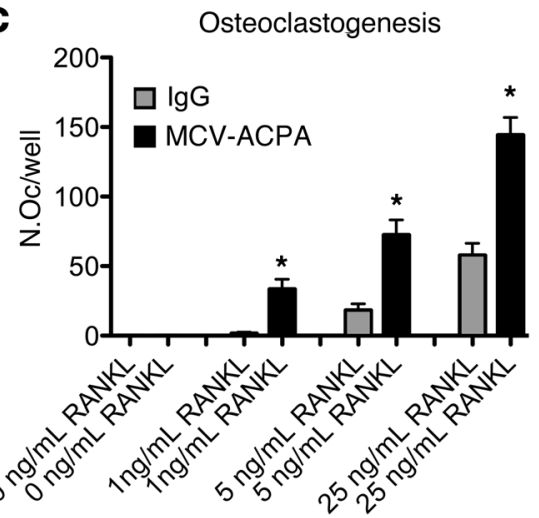

TNF- $\alpha$

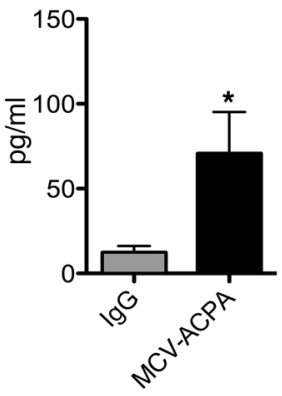

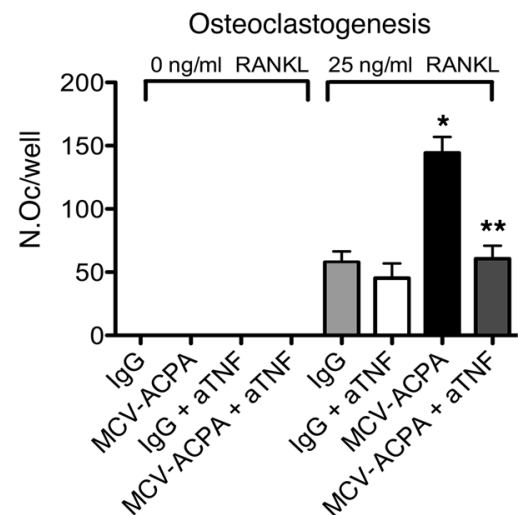

Figure 6

Bone loss elicited by MCV-ACPAs is induced by TNF-mediated increase of osteoclast precursor trafficking and differentiation. (A) Serum levels ( $n=5$ per group) of CTXI, osteocalcin, and TNF- $\alpha$ in Rag $1^{-/-}$mice that were left untreated or treated with control IgG or MCV-ACPAs. (B) FACS analysis ( $n=5$ per group) of spleen cells for the number of $C D 11 \mathrm{~b}+C D 14+$ cells (osteoclast precursors) and their expression of CD115 and RANK. (C) Osteoclastogenesis assay ( $n=3$ per group) of spleen cells from Rag $1^{-/-}$mice treated with control IgG or MCV-ACPAs with various concentrations of RANKL. TNF- $\alpha$ release and osteoclastogenesis assays ( $n=3$ per group) from spleen cells of wild-type mice treated with control lgG or MCV-ACPAs are also shown. aTNF, blockade of TNF- $\alpha$ by $100 \mathrm{ng} / \mathrm{ml}$ etanercept. ${ }^{*} P<0.05$.

challenged mice. In contrast, serum levels of osteocalcin, a marker of bone formation, were not affected (Figure 6A). Interestingly, we also found an increase in systemic levels of TNF- $\alpha$ in mice exposed to ACPAs (Figure 6A), although we did not find signs of arthritis, induction of which may depend on a specific susceptible genetic background, as reported previously (20). TNF- $\alpha$, however, is also a major inducer of osteoclastogenesis by enhancing differentiation of osteoclasts and by trafficking of osteoclast precursors $(21,22)$.

Indeed, when assessing the spleens of ACPA-challenged mice, we found a significant increase of $\mathrm{CD} 11 \mathrm{~b}^{+} \mathrm{CD} 14^{+}$osteoclast precur- sors (Figure 6B), which has been previously shown as an effect of TNF- $\alpha$ on cells of the osteoclast lineage (22). Moreover, the cells expressed higher surface levels of the MSCF receptor CD115 (also known as c-Fms) and the RANKL receptor RANK, making them more susceptible to further differentiation into mature osteoclasts (Figure 6B). Accordingly, spleen cells from ACPA-challenged mice showed overall higher osteoclastogenic potential by being more sensitive to stimulation by small amounts of RANKL, as well as allowing differentiation of higher numbers of osteoclasts (Figure $6 \mathrm{C})$. These in vivo results were confirmed by further in vitro obser- 
vations: exposure to MCV-ACPAs elicited TNF- $\alpha$ release from spleen cells of untreated wild-type mice and enhanced osteoclastogenesis in the presence of MCSF and RANKL, an effect that was blocked by TNF- $\alpha$ inhibition (Figure 6C).

\section{Discussion}

The present study provides a link between immune system and bone. We showed that autoantibodies against citrullinated vimentin (i.e., MCV-ACPAs), which are specifically found in patients with $\mathrm{RA}$, directly led to osteoclast differentiation and bone resorption in vitro and in vivo. RA is one of the most common autoimmune diseases, affecting $1 \%$ of the worldwide population, and a major trigger for local and systemic bone loss, resulting in joint destruction and increased fracture risk. More than two-thirds of patients with RA develop immune responses against citrullinated proteins, which is highly specific for RA (3). Despite being known for many years, it has been always unclear why these autoantibodies are associated with a more severe disease course, in particular more destructive joint disease. Although the direct link we have demonstrated between autoantibodies and bone loss may not be the only explanation for the more severe disease course of ACPA-positive patients (yet-unidentified differences in the gestalt of inflammation between ACPA-positive and -negative patients cannot be ruled out completely), these data provide an attractive concept of how the breakdown of immune tolerance can damage our skeletal system.

Current concepts suggest a finely balanced interplay between the immune system and bone. These concepts have been driven by clinical observation of bone destruction in RA and other inflammatory diseases. Activation of the innate immune system and production of proinflammatory cytokines, in particular TNF, triggers bone loss by inducing the expression of RANKL, a key osteoclastogenic molecule (21). Moreover, activation of the adaptive immune system, in particular T cell activation, also supports bone destruction. $\mathrm{T}$ cell activation, particularly that of the proinflammatory TH17 subset, fosters the expression of RANKL and induces osteoclastogenesis (23). These processes also explain why autoimmune inflammatory diseases such as RA are linked to bone loss.

Although not all RA patients experience bone destruction, others face rapid bone loss and destruction of the joints leading to crippling and functional loss. The clinical heterogeneity of RA with respect to joint damage suggests that there are additional factors that determine bone destruction in patients with arthritis. In fact, the strongest risk factor is the presence of ACPAs. Despite this strong clinical association, the role of autoantibodies on bone has so far been largely neglected. The potential principle that autoantibodies modify bone has been shown in rare cases of autoantibodies against the decoy receptor of RANKL, osteoprotegerin, which caused bone loss in patents with celiac diseases (24). However, so far none of the classical (i.e., frequently observed) autoantibodies has been mechanistically linked to bone loss.

Our study directly links ACPA, a common autoantibody prevalent in RA patients worldwide, to bone loss. ACPA binding to citrullinated vimentin enhanced the differentiation of osteoclast precursors into mature bone resorbing cells in vitro and in vivo. As a consequence, ACPAs enhanced bone resorption in vitro and in vivo and elicited bone loss in vivo by increasing osteoclast differentiation. Interestingly, the mechanism by which ACPAs trigger bone loss involved TNF- $\alpha$, the pivotal cytokine in the pathogenesis of RA: MCV-ACPAs induced production of TNF- $\alpha$ by osteoclast precursors, extending previous observations that ACPAs can directly affect cells of the monocyte/macrophage lineage (25-28). For bone, TNF- $\alpha$ is highly important, as it not only facilitates the trafficking of osteoclast precursors from the bone marrow to the lymphoid organs, but also renders them more susceptible to further differentiation into osteoclasts by inducing the receptors of key differentiation factors for osteoclasts, such as MSCF and RANKL. The net effect of ACPAs is thus a TNF-mediated induction of osteoclastogenesis and bone resorption. Although vimentin affects osteoblast differentiation (29), we could not detect relevant citrullination of vimentin in osteoblasts, and ACPAs also did not affect osteoblast differentiation and bone formation in vitro. We conclude that ACPAs, which are known to emerge years ahead of the clinical onset of RA (30), not only increase susceptibility to RA development, but also appear to disturb bone balance by making individuals more prone to bone resorption. This concept also serves to explain why RA patients already show low bone mass at the onset of disease (31) and why bone damage in RA patients with ACPAs is far higher compared with those without ACPAs.

The observation that osteoclasts contained large amounts of citrullinated vimentin also sheds light on the role of posttranslational modifications, in particular citrullination, on the differentiation of cells of the monocyte lineage. Previous studies have shown that PAD2 and PAD4 are the predominant isoforms expressed in monocytes (32). We observed here that osteoclast precursors shared a similar PAD expression pattern, with PAD2 and PAD4 as the dominant PAD isoforms. In contrast, cells of the osteoclast lineage did not express PAD1 and PAD3. This expression pattern of PADs in osteoclasts was in fact similar to the one found in the inflamed synovial tissue of RA patients $(33,34)$. Furthermore, during the process of osteoclast differentiation, the PAD expression pattern of cells shifted from PAD4 to PAD2. This observation suggests that PAD2 is the major contributor to protein citrullination in osteoclasts. Interestingly, previous studies have shown that monocyte differentiation into macrophages also induces a shift from PAD4 to PAD2 expression, similar to what we found in osteoclasts (32, $35)$. In contrast, PAD4 has been recently shown to function as an antimicrobial component forming extracellular traps (36). These data indicate that PAD expression and the citrullination pattern of proteins may differ among the various leukocytes subsets and allow for different functional properties of the individual subsets.

Remarkably, calcium has been shown to be a key inducer of PAD, and the calcium-rich environment to which osteoclasts are exposed may foster PAD expression and protein citrullination (37). Indeed, a strong increase in the citrullination of vimentin was observed during osteoclast differentiation, and citrullination occurred at the same region of citrullinated vimentin, which is recognized by the human autoantibody. Vimentin, in fact, has been identified as an important component of the adhesion zone of the osteoclast (38), and it remains to be determined whether its citrullination affects cell function and/or survival. Macrophages, for instance, can secrete vimentin, which facilitates bacterial killing and oxidation product synthesis (39).

In summary, our data showed a disease-relevant link among autoantibody formation, autoimmune disease, and bone loss in humans, which we believe to be novel. Autoantibodies against citrullinated vimentin bind to cells of the osteoclast lineage, foster their differentiation into bone resorbing cells, and promote bone loss. Our data extended current concepts on the interactions of immune system, inflammation, and bone by directly linking ACPA, a key autoantibody in human inflammatory disease, with 
bone loss, leading to joint destruction and functional decline in arthritis. Thus, our findings suggest a link between autoimmunity and the skeleton in human disease.

\section{Methods}

Purification of $M C V-A C P A s$. Samples of serum with very high ACPA response ( $>1,000 \mathrm{IU} / \mathrm{ml}$ ) were obtained from $3 \mathrm{RA}$ patients presenting at the Department of Medicine 3 of the University Clinic of Erlangen. ACPA activity was detected with a commercially available ELISA for detection of antibodies against citrullinated vimentin (Orgentec Diagnostika).

Recombinant MCV was conjugated to CNBr-sepharose (GE Healthcare) (1 $\mathrm{mg}$ protein/ml equilibrated sepharose) according to the manufacturer's instructions and stored at $4{ }^{\circ} \mathrm{C}$ in PBS with $0.1 \%$ BSA. Prior to affinity purification of ACPAs, IgG was purified by affinity chromatography on a protein A column (GE Healthcare). Eluted IgG fractions were immediately neutralized with 1:10 volume of $1 \mathrm{M}$ Tris-buffer ( $\mathrm{pH}$ 8.0) and dialyzed against PBS ( $\mathrm{pH}$ 7.2). Each purified IgG fraction was applied to a separate citrullinated vimentin affinity column ( $1 \mathrm{ml}$ matrix per serum sample) and incubated for 2 hours at $4{ }^{\circ} \mathrm{C}$, rotating end over end. The column was washed with $0.5 \mathrm{M}$ $\mathrm{NaCl}$ with $0.05 \%$ Tween 20 in PBS (pH 7.2), and the unbound fraction was collected. The bound anti-MCV antibodies (i.e., MCV-ACPAs) were eluted with $3.5 \mathrm{M} \mathrm{MgCl}_{2}$ ( $\mathrm{pH} 7.5$ ). The high salt buffer of the purified IgG fractions was exchanged against PBS using a desalting column (Zebra Spin; Pierce). MCV-ACPA concentration was increased by ultrafiltration on a Vivaspin $30 \mathrm{~K}$ unit (Millipore). The content of the MCV-ACPAs was verified with ELISA for measuring MCV autoantibodies (Orgentec Diagnostika). Additionally, the purification of the MCV-ACPAs and the successful cleavage with immobilized pepsin was verified with SDS-PAGE electrophoresis and staining with Coomassie blue.

Generation of Fab fragments. Immobilized pepsin allows efficient generation of $\mathrm{F}\left(\mathrm{ab}^{\prime}\right) 2$ fragments from IgG. Pepsin is a nonspecific endopeptidase that is active only at acidic $\mathrm{pH}$ and irreversibly denatured at neutral or alkaline $\mathrm{pH}$. Immobilized pepsin is advantageous because the digestion can be immediately stopped by simply removing the gel from the IgG. Digestion by pepsin normally produces a $\mathrm{F}\left(\mathrm{ab}^{\prime}\right) 2$ fragment and numerous small peptides of the Fc portion. The buffer of the MCV-ACPAs was exchanged against digestion buffer ( $0.1 \mathrm{M}$ sodium acetate; $\mathrm{pH} 4.5)$ with Zebra spin column. $1 \mathrm{ml}$ of this prepared IgG solution was added to a tube containing $100 \mu$ l equilibrated Immobilized Pepsin (catalog no. 20343; Pierce) and incubated in a thermomixer for 4 hours with constant mixing at 600 $\mathrm{rpm}$ at $37^{\circ} \mathrm{C}$. To separate digested protein from the Immobilized Pepsin, the solution was centrifuged at $1,000 \mathrm{~g}$ for 5 minutes. The supernatantcontaining digested protein was decanted into a new tube, and Protein A Columns (Pierce) were used to separate undigested IgG from $\mathrm{F}\left(\mathrm{ab}^{\prime}\right) 2$ fragments. To remove small Fc fragments, the eluted fractions were dialyzed and concentrated with ultrafiltration units (50K MWCO).

Analysis of antibody sialylation. Enzyme-linked lectin-specific ACPA assay (sialACPA ELISA) was used to analyze ACPA sialylation. Neuraminidase treatment was done to cleave selectively sialic acid-containing carbohydrates on the autoantibodies. Sialic acid release was induced by digestion at $25^{\circ} \mathrm{C}$ for 2 hours with $50 \mathrm{mU}$ neuraminidase cloned from Clostridium perfringens and overexpressed in E. coli (NEB) in $200 \mu \mathrm{l}$ of $50 \mu \mathrm{M}$ sodium citrate ( $\mathrm{pH}$ 6.0).

Synthesis of MCV peptides. Previously published sequences of MCV (17) were used as a template to synthesize overlapping 17-mer peptides, in accordance with Fmoc-chemistry at the Peptide Specialty of Perbio Mimotopes (Perbio Science Deutschland GmbH). Each peptide had a 12-amino acid residue overlap with the neighboring peptide. The synthesis through the MCV amino acid sequence resulted in 91 peptides with the general formula Biotin-SGSG-PEPTIDE-Amide. The N-terminal extension of the peptides (Biotin-SGSG) was designed for defined flexibility and to incorporate an affinity tag. Each arginine-containing peptide was synthesized as the citrulline residue variant. Crude fractions after peptide synthesis were purified using high-performance liquid chromatography. Quality and purity of the peptide was assessed by mass spectrometry and analytical high-performance liquid chromatography according to the manufacture's instructions (Perbio Science Deutschland GmbH).

Epitope characterization by peptide ELISA. Microtiter plates (Maxisorb) were precoated with $1 \mu \mathrm{g} / \mathrm{ml}$ streptavidin (Perbio Science Deutschland GmbH) in PBS ( $\mathrm{pH}$ 7.6). After incubation for 2 hours at $25^{\circ} \mathrm{C}$, plates were blocked with $1 \%$ BSA in PBS for 30 minutes at room temperature. Theses plates were used for binding of the biotinylated peptides/antigens. The biotinylated 17 -mer peptides at a concentration of $0.5 \mu \mathrm{g} / \mathrm{ml}$ were also diluted in PBS ( $\mathrm{pH} 7.6)$ and incubated overnight at $4{ }^{\circ} \mathrm{C}(100 \mu \mathrm{l} /$ well). In addition to the 91 peptides, biotinylated recombinant $\mathrm{MCV}$, protein $\mathrm{A}$, and rheumatoid factor antigen were used as internal control antigens in the available 5 cavities of a 96-well microtiter plate. Coating and blocking was done as described for peptides. Possible excesses of peptides/antigens were eliminated by washing the cavities with $200 \mu \mathrm{l} /$ well 0.1\% Tween 20 in PBS. Finally, flicking and slapping removed any residual solution. The assay was performed in accordance with the general protocol for the Orgentec ELISA system. In brief, the serum samples were diluted 1:101 in sample buffer (PBS containing BSA and Tween), added to the wells, and then incubated for 30 minutes $(100 \mu \mathrm{l} /$ well). After 3 washing steps $(300 \mu \mathrm{l} /$ well), horseradish peroxidase-conjugated anti-human IgG (Dianova) was added and incubated for 30 minutes $(100 \mu \mathrm{l} /$ well). Visualization was done by incubation with 3,3',5,5'-tetra-methyl benzidine substrate for 15 minutes (100 $\mu \mathrm{l} /$ well), and the reaction was terminated by adding $50 \mu \mathrm{l} \mathrm{stop} \mathrm{solution}(0.5 \mathrm{~mol} / \mathrm{l}$ $\mathrm{H}_{2} \mathrm{SO}_{4}$ ) to each well. Finally, absorbance at $450 \mathrm{~nm}$ was determined using an ELISA reader (Rainbow Reader; Tecan). All steps were carried out at room temperature. Background $\mathrm{OD}$ was obtained by adding each serum to a well without protein. A positive serum was defined by an $O D$ value more than twice that of background OD (40). Each serum sample was tested in duplicate and to further define the assay characteristics.

Reactivity to citrullinated and noncitrullinated peptides. Citrullin- and respective arginine-containing peptides (denoted by bold X and R, respectively) of vimentin (normal, SAVRARSSVPGVRK; citrullinated, SAVRAXSSVPGVRK), fillagrin (normal, SHQESTRGRSRGRSGRSGS; citrullinated, SHQESTXGXSXGRSGRSGS), and collagen (normal, ARGLTGRPGDAK; citrullinated, AXGLTGXPGDAK) as well as fibrinogen-derived peptides (normal, RPAPPPISGGGYRAR; citrullinated, RPAPPPISGGGYXAX) were synthesized by solid-phase peptide synthesis (FMOC strategy) in the Department of Peptide Chemistry of Eötvös Loránd University (Budapest, Hungary). Biotinylated peptides were bound to NeutrAvidin-precoated $(5 \mu \mathrm{g} / \mathrm{ml})$ plates. After adding biotinylated peptides $(1 \mu \mathrm{g} / \mathrm{ml})$, the wells were blocked ( $40 \mathrm{mM}$ Tris- $\mathrm{HCl}, 150 \mathrm{mM} \mathrm{NaCl}, 0.1 \%$ Tween, $0.5 \% \mathrm{BSA}$ ), sera samples were added (1:400 or 1:100), and then plates were incubated for 1 hour at $37^{\circ} \mathrm{C}$. After washing, rabbit anti-human IgG conjugated to horseradish peroxidase was added at 1:2,000 dilution for 1 hour at $37^{\circ} \mathrm{C}$. The signal was developed by tetramethylbenzidin $(10 \mathrm{mg} / \mathrm{ml}$, diluted in 0.1 $\mathrm{M}$ acetate buffer [ $\mathrm{pH}$ 5.5] and $30 \% \mathrm{H}_{2} \mathrm{O}_{2}$ ) for about 5 minutes at room temperature. The reaction was stopped by $4 \mathrm{~N} \mathrm{H}_{2} \mathrm{SO}_{4}$. Absorbance was measured at $450 \mathrm{~nm}$ (correction wavelength set at $620 \mathrm{~nm}$ ) using a Labsystems Multiskan MS spectrophotometer. To analyze our results, an OD ratio (OD of citrullin-containing peptide minus $\mathrm{OD}$ of arginine-containing peptide) was calculated and compared between various groups (Kruskal-Wallis test).

Mass spectrometry of citrullinated proteins in osteoclast lineage cells. Cell pellets $\left(1 \times 10^{6}\right.$ cells each) were suspended in $400 \mu l$ lysis buffer containing $0.1 \%$ RapiGest in $50 \mathrm{mM}$ ammonium bicarbonate and protease inhibitor cocktail (Complete Mini; Roche Diagnostics). The homogenate was sonicated using 
a probe tip sonicator (power 50\%; Bandelin Sonopuls, Buch \& Holm) 3 times for 20 seconds on ice. The homogenate was centrifuged at $9,000 \mathrm{~g}$ at $4{ }^{\circ} \mathrm{C}$ for 5 minutes, and the pellet was discarded. Prepacked PD SpinTrap columns (GE Healthcare) were used for desalting and buffer exchange against $50 \mathrm{mM}$ ammonium bicarbonate. The protein concentration was measured using a NanoDrop spectrophotometer (Thermo Scientific). $100 \mu \mathrm{g}$ protein from each cell sample was reduced in $200 \mathrm{mM}$ dithiothreitol at $56^{\circ} \mathrm{C}$ for 30 minutes followed by alkylation by $1 \mathrm{M}$ indoleacetic acid for 1 hour at room temperature in darkness. Endoproteinase Lys-C sequencing grade (Roche Diagnostics $\mathrm{GmbH}$ ) was added at 1:50 enzyme/substrate, and samples were incubated at $37^{\circ} \mathrm{C}$ overnight. After digestion, the samples were transferred to a Pall Nanosep centrifugal device with Omega membrane MWCO 10 kDa (Pall Corp.). Peptides were cleaned up by ZipTip pipette tips containing $\mathrm{C}_{18}$ media (Millipore) and dried using speed-vac prior to mass spectrometry analysis.

Samples were resuspended in $0.1 \%$ formic acid and $1 \mu \mathrm{g}$ of each was injected onto a Waters NanoAquity UPLC equipped with a nanoAquity 5- $\mu \mathrm{m}$ TRAP C18 $180-\mu \mathrm{m}$ i.d. $\times 20-\mathrm{mm}$ trapping column and a $1.7-\mu \mathrm{m}$ BEH130 C18 75- $\mu$ m i.d. $\times 150-\mathrm{mm}$ reversed-phase analytical column. The mobile phase consisted of (a) $0.1 \%$ formic acid in water and (b) $0.1 \%$ formic acid in acetonitrile. The peptides were separated using a gradient of $3 \%-40 \%$ of the latter for the first 90 minutes, then $40 \%-90 \%$ of the latter again for the following 8 minutes. The peptides were analyzed using a Waters Q-Tof Premier mass spectrometer operated in data-dependent mode with 4 components, each acquired for $0.9 \mathrm{~s} /$ spectra, $1-3$ spectra/peptide. High mass accuracy was achieved by lock-spray using Glu-1-Fibrinopeptide. The raw data were processed using Mascot Distller version 2.4.0 into .mgf files.

Peptide and protein identification was performed using X!Tandem open source software. The searches were performed against the human SwissProt database, with Lys-C specificity and semi-style cleavage allowed. Mass deviations for precursor ions and for fragment ions were set to $20 \mathrm{ppm}$ and $0.5 \mathrm{Da}$, respectively. Carbamidomethylation was chosen as a fixed modification, while citrullination (R), deamidation ( $\mathrm{N}, \mathrm{Q})$, and oxidation $(\mathrm{M})$ were set as variable modifications. Only proteins with expectation value $\log _{\mathrm{e}}$ below -3 were considered as a positive match.

Osteoclast cultures and resorption tests. Peripheral blood mononuclear cells (PBMCs) were isolated from $10 \mathrm{ml}$ EDTA-blood of healthy donors using a Ficoll gradient (Lyphoflot; BioRad) and incubated in 48-well plates ( $7.5 \times 10^{5}$ cells/well) in $\alpha$-MEM with $1 \%$ PenStrep (both Gibco, Invitrogen). After 3 hours, cells were washed for monocyte enrichment and cultivated for 14 days in $\alpha$-MEM supplemented with $10 \%$ heat-inactivated fetal BSA (Biochrom AG), 1\% penicillin/streptomycin (Gibco, Invitrogen), $10 \mathrm{ng} / \mathrm{ml} \mathrm{MCSF}$, and submaximal concentrations of $1 \mathrm{ng} / \mathrm{ml}$ RANKL (both Peprotech). Every 3 days, medium was replaced with fresh medium. Various concentrations of ACPA-positive serum or MCV-ACPAs were added as indicated. Osteoclast differentiation was evaluated by staining cells for TRAP using a Leukocyte Acid Phosphatase Kit (Sigma-Aldrich) according to the manufacturer's instructions. For global bone resorption, the same protocol was performed on bovine bone slides, and resorption pit area was assessed. For assessment of bone resorption per single osteoclast (resorption index), osteoclasts were detached from plastic plates using acutase (A6964; Sigma-Aldrich) and replated on bone slices after 4 days of culture. After 48 hours, osteoclasts were scraped from the slices using cotton buds, and slices were stained for 30 seconds in a $1 \%$ toluidin solution. Acquisition of images was done on a widefield microscope with side illumination, which identified resorption pits by their dark color and 1-sided shining ("dawn on the valley" effect).

Immunofluorescence. Osteoclasts were fixed with $4 \% \mathrm{PFA}(\mathrm{pH} 7.2$ ) in PBS for 10 minutes at $37^{\circ} \mathrm{C}$. Fixed samples were then washed 3 times in PBS and permeabilized for 10 minutes with $0.2 \%$ Triton X-100 in PBS. Next, samples were preblocked for 10 minutes with a solution of $2 \%$ BSA, $0.1 \%$ Triton X-100, and $0.1 \%$ azide in PBS. All remaining washing steps and antibody dilutions were done using this solution. Samples were incubated with 1:50 dilution of MCV-ACPA, Alexa Fluor 488 mouse anti-human vimentin antibody, or Alexa Fluor 488 phalloidin (all Sigma-Aldrich). For detection of bound MCVACPA, either Alexa Fluor 488- or Alexa Fluor 647-conjugated anti-human IgG antibody was used. Cells were then incubated with DAPI $(10 \mu \mathrm{g} / \mathrm{ml})$ and mounted with FluorSave reagent (Calbiochem). Fluorescence images were acquired with a Zeiss spinning disk confocal microscope.

Immunoblotting. PBMCs $\left(7.5 \times 10^{5}\right.$ cells/well $)$ were cultivated in 48 -well plates with MCSF, RANKL and TGF- $\beta$ for 14 days as indicated above. Cells were lysed in Laemmli buffer (BioRad) either directly after the isolation or after various times of culture. Proteins were separated by electrophoresis in $10 \%$ sodium dodecyl sulfate-polyacrylamide gels and blotted onto polyvinylidene difluoride membranes (Millipore). After blocking with $5 \%$ dry milk prepared in PBS containing $0.1 \%$ Tween 20 , the membranes were subjected to immunoblotting. Antibodies used were as follows: rabbit anti- $\beta$-actin antibody (Sigma-Aldrich), chicken anti-citrullinated vimentin antibody, purified human MCV autoantibody (i.e., MCV-ACPAs), rabbit anti-PAD2 antibody, rabbit anti-PAD4 antibody, mouse anti-vimentin antibody (all Abcam). Detection was done by respective peroxidase-conjugated antibodies (all Promega) and chemoluminescence reagent (ECL; Thermo Scientific).

Quantitative real-time PCR analysis. PBMCs $\left(7.5 \times 10^{5}\right.$ cells/well $)$ were cultivated in 48-well plates with MCSF, RANKL, and TGF- $\beta$ for 14 days. Directly after the isolation or after various times of culture, total RNA was extracted using peqGOLD TriFast (Peqlab) according to the manufacturer's instructions. RNA was transcribed into cDNA using an oligo(dT) primer and MuLV reverse transcriptase (Roche). Quantitative real-time PCR was performed using SYBR Green I-dTTP (Eurogentec) using the following primer pairs: $\beta_{2}$-microglobulin $\left(\beta_{2}-\mathrm{MG}\right)$ forward, GATGAGTATGCCTGCCGTGTG; $\beta_{2}$-MG reverse, CAATCCAAATGCGGCATCT; PAD2 forward, AGCTCTACCATGTGGTCAAGT; PAD2 reverse, GGTGTCCGTGAAGATGGGAG; PAD4 forward, CCGGCAAAGTGAAGCCAAC; PAD4 reverse, CTCAGGGTCATCAGCGACA. Each sample was analyzed in duplicate and normalized to the level of $\beta_{2}-\mathrm{MG}$.

Serum parameters. In human serum samples, we measured CTXI using a commercial kit (Immundiagnostic Systems) based on ELISA. TRAP5b (Quidel) and cathepsin K (Biomedica) were also measured by ELISA. ACPAs were measured by commercial ELISA (Phadia/Thermofisher Scientific), rheumatoid factor by nephelometry (Beckmann Coulter). In murine serum samples, CTXI (Ratlaps; Nordic Bioscience), osteoclacin (Quidel), and TNF- $\alpha(\mathrm{R} \& \mathrm{D})$ were measured by commercial ELISAs.

$\mu C T$. 10 female Rag1 $1^{-/}$mice were injected i.p. with $50 \mu \mathrm{g}$ lipopolysaccharide and with $500 \mu \mathrm{g}$ normal human IgG, $500 \mu \mathrm{g}$ MCV-ACPAs, or PBS. After 4 weeks, mice were sacrificed. The right tibia was prepared and stored in $70 \%$ ethanol for $\mu \mathrm{CT}$ analysis (41). $\mu \mathrm{CT}$ analyses were done with a commercially available desktop $\mu \mathrm{CT}$ ( $\mu$ CT35; SCANCO Medical AG) (42). The following acquisition parameters were used: voltage, $40 \mathrm{kV}$; X-ray current, $250 \mu \mathrm{A}$; exposure time, $5,000 \mathrm{~ms} /$ projection for 720 projections; matrix, $1024 \times 1024$; voxel size in reconstructed image, $9 \mu \mathrm{m}$. Images were analyzed using a plugin programmed for Amira 4.1.2 (Mercury) for histomorphometric parameters at the metaphyses of the proximal tibiae: ratio of bone volume to total volume, trabecular number, and trabecular thickness (41).

Mouse histology. Mice were treated as described above, and the left tibia was used for histology Bones were fixed overnight in $4 \%$ formalin and decalcified in EDTA (Sigma-Aldrich) until they were pliable. Serial paraffin sections $(2 \mu \mathrm{m})$ were stained for TRAP for osteoclast detection. All analyses were performed using a microscope (Nikon) equipped with a digital camera and an image analysis system for performing histomorphometry (Osteomeasure; OsteoMetrics). 
FACS and murine osteoclast assays. For FACS staining, $1 \times 10^{6}$ murine spleen cells were washed with $1 \mathrm{ml}$ PBS containing $0.1 \%$ BSA resuspended in $100 \mu \mathrm{l} \mathrm{FACS}$ buffer and incubated with saturating amounts of PECy7-, FITC-, biotin-, or PE-labeled antibodies against CD11b, CD14 (both BD Bioscience), CD115 (c-Fms, MCSF receptor; Biolegends), and RANK (CD265; Abcam) for 30 minutes at $4^{\circ} \mathrm{C}$ in the dark. Cells were also stained with isotype control antibody. A FACSCanto Flow Cytometer (BD Bioscience) was used for analysis. For osteoclast assays, bone marrow was isolated by flushing femoral bones with complete $\alpha$ DMEM. Osteoclast precursors were then isolated from bone marrow-derived cell suspensions using CD11b microbeads (Miltenyi Biotec) according to the manufacturer's instructions. The purity of isolated monocytes was assessed by flow cytometry analysis using FITC-labeled antibodies against CD11b (Miltenyi Biotec). CD $11 \mathrm{~b}^{+}$monocytes were plated in 96 -well plates $\left(2.5 \times 10^{5}\right.$ cells/well) or 48-well plates $\left(5 \times 10^{5}\right.$ cells/well $)$ in $\alpha$ MEM supplemented with $10 \%$ fetal calf serum, $30 \mathrm{ng} / \mathrm{ml} \mathrm{M}$-CSF, and various doses $(0,1,5$, or $25 \mathrm{ng} / \mathrm{ml}$ ) of RANKL (all R\&D Systems). To examine the contribution of ACPAs to osteoclast development, MCV-ACPAs $(10 \mathrm{ng} / \mathrm{ml})$ were added to the cultures. Medium was changed after 72 hours. To examine the contribution of TNF- $\alpha$ blocking, experiments with $100 \mathrm{ng} / \mathrm{ml}$ etanercept, a TNF receptor $2-\mathrm{Fc}$ fusion protein that also inhibits murine TNF- $\alpha$, were performed. Osteoclast differentiation was evaluated by staining cells for TRAP using a Leukocyte Acid Phosphatase Kit (Sigma-Aldrich) according to the manufacturer's instructions.

Statistics. For comparison of multiple groups, 1-way ANOVA with Bonferroni correction was used in case values showed Gaussian distribution. In the absence of Gaussian distribution, the Kruskal-Wallis test with Dunn's correction was performed. A $P$ value less than 0.05 was considered significant.
Study approval. Studies in animals and humans were reviewed and approved by the ethics committee of the University Clinic of Erlangen. All subjects provided informed consent prior to their participation in the study.

\section{Acknowledgments}

We thank Fruzsina Babos and Anna Magyar (HAS-ELTE Research Group of Peptide Chemistry, Eötvös Loránd University, Budapest, Hungary) for peptide synthesis, as well as Marlène Gallet (Institut de Génomique Fonctionnelle de Lyon, Lyon, France) for valuable input on the bone resorption assay. This study was supported by the Deutsche Forschungsgemeinschaft (FG 661/TP4 and SPP1468IMMUNOBONE), the Bundesministerium für Bildung und Forschung (BMBF; project ANCYLOSS), the MASTERSWITCH project of the European Union, the IMI-funded project BTCure, and Agence de Recherche contra le Cancer (ARC). G. Sarmay and E. Szarka are supported by the Hungarian National Development Agency and OTKA (CK80689). U. Harre is a member of the Graduiertenkolleg 1660. D. Georgess is an Early Scientific Researcher recipient from a Marie Curie action (T3Net project). The authors acknowledge the contribution of the PLATIM platform of UMS344/US8 Biosciences.

Received for publication September 12, 2011, and accepted in revised form March 7, 2012.

Address correspondence to: Georg Schett, Department of Internal Medicine 3, Rheumatology and Immunology, University of Erlangen-Nuremberg, Krankenhausstrasse 12, Erlangen D-91054, Germany. Phone: 49.9131.85.33363; Fax: 49.9131.85.34770; E-mail: georg.schett@uk-erlangen.de.
1. Firestein GS. Evolving concepts of rheumatoid arthritis. Nature. 2003;423(6937):356-361.

2. McInnes IB, Schett G. Cytokines in the pathogenesis of rheumatoid arthritis. Nat Rev Immunol. 2007;7(6):429-442.

3. Klareskog L, et al. A new model for an etiology of rheumatoid arthritis: smoking may trigger HLADR (shared epitope)-restricted immune reactions to autoantigens modified by citrullination. Arthritis Rheum. 2006;54(1):38-46.

4. Mahdi H, et al. Specific interaction between genotype, smoking and autoimmunity to citrullinated alpha-enolase in the etiology of rheumatoid arthritis. Nat Genet. 2009;41(12):1319-1324.

5. Vossenaar ER, Zendman AJ, van Venrooij WJ, Pruijn GJ. PAD, a growing family of citrullinating enzymes: genes, features and involvement in disease. Bioessays. 2003;25(11):1106-1118.

6. Wegner N, et al. Peptidylarginine deiminase from Porphyromonas gingivalis citrullinates human fibrinogen and $\alpha$-enolase: implications for autoimmunity in rheumatoid arthritis. Arthritis Rheum. 2010;62(9):2662-2672.

7. Vincent C, de Keyser F, Masson-Bessiere C, Sebbag M, Veys E, Serre G. Anti-perinuclear factor compared with the so called "antikeratin" antibodies and antibodies to human epidermis filaggrin, in the diagnosis of arthritides. Ann Rheum Dis. 1999;58(1):42-48.

8. De Rycke L, et al. Rheumatoid factor and anticitrullinated protein antibodies in rheumatoid arthritis: diagnostic value, associations with radiological progression rate, and extra-articular manifestations. Ann Rheum Dis. 2004;63(12):1587-1593.

9. van der Woude D, et al. Epitope spreading of the anti-citrullinated protein antibody response occurs before disease onset and is associated with the disease course of early arthritis. Ann Rheum Dis. 2010;69(8):1554-1561.
10. Schett G, Teitelbaum SL. Osteoclasts and arthritis. J Bone Miner Res. 2009;24(7):1142-1146.

11. Schett G, et al. High-sensitivity C-reactive protein and risk of nontraumatic fractures in the Bruneck study. Arch Intern Med. 2006;166(22):2495-2501.

12. Takayanagi H. Osteoimmunology: shared mechanisms and crosstalk between the immune and bone systems. Nat Rev Immunol. 2007;7(4):292-304.

13. van Gaalen FA, et al. Autoantibodies to cyclic citrullinated peptides predict progression to rheumatoid arthritis in patients with undifferentiated arthritis: a prospective cohort study. Arthritis Rheum. 2004;50(3):709-715.

14. Syversen SW, et al. Prediction of radiographic progression in rheumatoid arthritis and the role of antibodies against mutated citrullinated vimentin: results from a 10-year prospective study. Ann Rheum Dis. 2010;69(2):345-351.

15. van der Linden MP, et al. Value of anti-modified citrullinated vimentin and third-generation anticyclic citrullinated peptide compared with secondgeneration anti-cyclic citrullinated peptide and rheumatoid factor in predicting disease outcome in undifferentiated arthritis and rheumatoid arthritis. Arthritis Rheum. 2009;60(8):2232-2241.

16. Raza K, Mathsson L, Buckley CD, Filer A, Rönnelid J. Anti-modified citrullinated vimentin (MCV) antibodies in patients with very early synovitis. Ann Rheum Dis. 2010;69(3):627-628.

17. Bang $\mathrm{H}$, et al. Mutation and citrullination modifies vimentin to a novel autoantigen for rheumatoid arthritis. Arthritis Rheum. 2007;56(8):2503-2511.

18. Mathsson L, et al. Antibodies against citrullinated vimentin in rheumatoid arthritis: higher sensitivity and extended prognostic value concerning future radiographic progression as compared with antibodies against cyclic citrullinated peptides. Arthritis Rheum. 2008;58(1):36-45.

19. Dellagi K, Vainchenker W, Vinci G, Paulin D, Brou- et JC. Alteration of vimentin intermediate filament expression during differentiation of human hemopoietic cells. EMBO J. 1983;2(9):1509-1514.

20. Kuhn KA, et al. Antibodies against citrullinated proteins enhance tissue injury in experimental autoimmune arthritis. J Clin Invest. 2006;116(4):961-973.

21. Lam J, Takeshita S, Barker JE, Kanagawa O, Ross FP, Teitelbaum SL. TNF-alpha induces osteoclastogenesis by direct stimulation of macrophages exposed to permissive levels of RANK ligand. J Clin Invest. 2000;106(12):1481-1488.

22. Li P, et al. RANK signaling is not required for TNF-mediated increase in CD11(hi) osteoclast precursors but is essential for mature osteoclast formation in TNFalpha-mediated inflammatory arthritis. J Bone Miner Res. 2004;19(2):207-213.

23. Sato K, et al. Th17 functions as an osteoclastogenic helper $\mathrm{T}$ cell subset that links $\mathrm{T}$ cell activation and bone destruction. J Exp Med. 2006; 203(12):2673-2682.

24. Riches PL, McRorie E, Fraser WD, Determann C, van't Hof R, Ralston SH. Osteoporosis associated with neutralizing autoantibodies against osteoprotegerin. NEngl J Med. 2009;361(15):1459-1465.

25. Clavel C, et al. Induction of macrophage secretion of tumor necrosis factor alpha through Fcgamma receptor IIa engagement by rheumatoid arthritis-specific autoantibodies to citrullinated proteins complexed with fibrinogen. Arthritis Rheum. 2008;58(3):678-688.

26. Laurent L, et al. Fc \{gamma\} receptor profile of monocytes and macrophages from rheumatoid arthritis patients and their response to immune complexes formed with autoantibodies to citrullinated proteins. Ann Rheum Dis. 2011;70(6):1052-1059.

27. Lu MC, Lai NS, Yu HC, Huang HB, Hsieh SC, Yu CL. Anti-citrullinated protein antibodies bind surfaceexpressed citrullinated Grp78 on monocyte/macrophages and stimulate tumor necrosis factor alpha 
production. Arthritis Rheum. 2010;62(5):1213-1223.

28. Sokolove J, Zhao X, Chandra PE, Robinson WH. Immune complexes containing citrullinated fibrinogen costimulate macrophages via Toll-like receptor 4 and Fc $\gamma$ receptor. Arthritis Rheum. 2011;63(1):53-62.

29. Lian N, Wang W, Li L, Elefteriou F, Yang X. Vimentin inhibits ATF4-mediated osteocalcin transcription and osteoblast differentiation. J Biol Chem. 2009;284(44):30518-30525.

30. Guler-Yuksel M, et al. Changes in hand and generalised bone mineral density in patients with recent-onset rheumatoid arthritis. Ann Rheum Dis. 2009;68(3):330-336.

31. Rantapää-Dahlqvist S, et al. Antibodies against cyclic citrullinated peptide and IgA rheumatoid factor predict the development of rheumatoid arthritis. Arthritis Rheum. 2003;48(10):2741-2749.

32. Vossenaar ER, et al. Expression and activity of citrullinating peptidylarginine deiminase enzymes in monocytes and macrophages. Ann Rheum Dis. 2004;63(4):373-381.

33. Foulquier C, et al. Peptidyl arginine deiminase type 2 (PAD-2) and PAD-4 but not PAD-1, PAD-3, and PAD- 6 are expressed in rheumatoid arthritis synovium in close association with tissue inflammation. Arthritis Rheum. 2007;56(11):3541-3553.

34. Chang $X$, et al. Localization of peptidylarginine deiminase 4 (PADI4) and citrullinated protein in synovial tissue of rheumatoid arthritis. Rheumatology (Oxford). 2005;44(1):40-50.

35. Hojo-Nakashima I, Sato R, Nakashima K, Hagiwara $\mathrm{T}$, Yamada M. Dynamic expression of peptidylarginine deiminase 2 in human monocytic leukaemia THP-1 cells during macrophage differentiation. J Biochem. 2009;146(4):471-479.

36. Li P, Li M, Lindberg MR, Kennett MJ, Xiong N, Wang Y. PAD4 is essential for antibacterial innate immunity mediated by neutrophil extracellular traps. J Exp Med. 2010;207(9):1853-1862.

37. Arita K, Hashimoto H, Shimizu T, Nakashima K, Yamada M, Sato M. Structural basis for $\mathrm{Ca}(2+)$ induced activation of human PAD4. Nat Struct Mol Biol. 2004;11(8):777-783

38. Marchisio PC, Cirillo D, Naldini L, Primavera MV,
Teti A, Zambonin-Zallone A. Cell-substratum interaction of cultured avian osteoclasts is mediated by specific adhesion structures. J Cell Biol. 1984;99(5):1696-1705.

39. Mor-Vaknin N, Punturieri A, Sitwala K, Markovitz DM. Vimentin is secreted by activated macrophages. Nat Cell Biol. 2003;5(1):59-63.

40. Auger I, Balandraud N, Rak J, Lambert N, Martin M, Roudier J. New autoantigens in rheumatoid arthritis (RA): screening 8268 protein arrays with sera from patients with RA. Ann Rheum Dis. 2009;68(4):591-594.

41. Bouxsein ML, Boyd SK, Christiansen BA, Guldberg RE, Jepsen KJ, Müller R. Guidelines for assessment of bone microstructure in rodents using micro-computed tomography. J Bone Miner Res. 2010;25(7):1468-1486.

42. Hildebrand T, Laib A, Muller R, Dequeker J, Ruegsegger P. Direct three-dimensional morphometric analysis of human cancellous bone: microstructural data from spine, femur, iliac crest, and calcaneus. J Bone Miner Res. 1999;14(7):1167-1174. 